\title{
Review
}

\section{Apoptosome: a platform for the activation of initiator caspases}

\author{
$Q \mathrm{Bao}^{1}$ and Y Shi ${ }^{*, 1}$
}

Apoptosome refers to the adaptor protein complex that mediates the activation of an initiator caspase at the onset of apoptosis. In mammalian cells, caspase-9, caspase-8, and caspase-2 rely on the apoptotic protease-activating factor 1 (Apaf-1)apoptosome, death-inducing signaling complex (DISC), and PIDDosome, respectively, for activation. In Drosophila, activation of the caspase-9 homolog Dronc requires assembly of an apoptosome comprised of Dark/Hac-1/Dapaf-1. In Caenorhabditis elegans, activation of the caspase CED-3 is facilitated by the CED-4-apoptosome. Recent biochemical and structural investigation revealed significant insights into the assembly and function of the various apoptosomes. Nonetheless, conclusive mechanisms by which the initiator caspases are activated by the apoptosomes remain elusive. Several models have been proposed to explain the activation process. The induced proximity model summarizes the general process of initiator caspase activation. The proximity-driven dimerization model describes how initiator caspases respond to induced proximity and offers an explanation for their activation. Regardless of how initiator caspases are activated, enhanced activity must be correlated with altered active site conformation. The induced conformation model posits that the activated conformation for the active site of a given initiator caspase is attained through direct interaction with the apoptosome or through homo-oligomerization facilitated by the apoptosome.

Cell Death and Differentiation (2007) 14, 56-65. doi:10.1038/sj.cdd.4402028; published online 15 September 2006

Apoptosis, the prevalent form of programmed cell death, plays a central role in the development and homeostasis of all multi cellular organisms. ${ }^{1}$ There are two well-characterized apoptotic pathways in mammalian cells: the extrinsic pathway and the intrinsic pathway. The extrinsic pathway, exemplified by the elimination of unwanted cells during animal development, is initiated by ligand-induced activation of the death receptors at the plasma membrane. ${ }^{2}$ The intrinsic cell death pathway, on the other hand, is triggered by cellular stress signals such as DNA damage. ${ }^{3}$ A molecular hallmark of apoptosis is the activation of caspases - specific proteases that execute cell death through cleavage of multiple protein substrates.

Apoptotic caspases constitute two general classes: initiator (or apical) caspase and effector (or executioner) caspase. ${ }^{4}$ The onset of apoptosis requires a cascade of sequential activation of initiator and effector caspases. ${ }^{4}$ Although the first caspase, interleukin $1 \beta$-converting enzyme (ICE; or caspase1 ), was identified in humans, the critical involvement of caspase in apoptosis was discovered in the nematode Caenorhabditis elegans, in which the indispensable gene ced-3 was found to encode a cysteine protease and to closely resemble the mammalian ICE. ${ }^{5,6}$ Since then, at least 14 distinct mammalian caspases have been identified, with 11 from the human genome. ${ }^{7}$ Seven mammalian caspases, including four initiator and three effector caspases, are known to play important roles in apoptosis (Figure 1). The functional homolog of mammalian caspase-9 is Dronc in Drosophila Melanogaster and CED-3 in C. elegans (Figure 1). It should also be noted that CED-3 is the only known apoptotic caspase in $C$. elegans, likely fulfilling a dual role of both initiator and effector caspase.

All caspases are produced in cells as catalytically inactive zymogens. The activation of an effector caspase, such as caspase-3 or caspase-7, is executed by an initiator caspase, such as caspase-9, through proteolytic cleavage after a specific internal Asp residue to separate the large and small subunits of the mature caspase (Figure 1). ${ }^{7}$ As a consequence of the intrachain cleavage, the catalytic activity of an effector caspase is enhanced by several orders of magnitude. ${ }^{8}$ Once activated, the effector caspases are responsible for the proteolytic degradation of a broad spectrum of cellular targets that ultimately lead to cell death.

In contrast to effector caspases, the activation of an initiator caspase and its regulation are quite complex. All initiator caspases are activated through autocatalytic intrachain cleavages. ${ }^{4,9}$ Although the intrachain cleavage is essential

\footnotetext{
${ }^{1}$ Department of Molecular Biology, Lewis Thomas Laboratory, Princeton University, Princeton, NJ, USA

${ }^{*}$ Corresponding author: Y Shi, Department of Molecular Biology, Lewis Thomas Laboratory, Princeton University, Washington Road, Princeton, NJ 08544, USA. Tel: 609-258-6071; Fax: 609-258-6730; E-mail: ygshi@ princeton.edu

Keywords: apoptosome; initiator caspase; apoptosis; DISC

Abbreviations: Apaf-1, apoptotic protease-activating factor 1; C. elegans, Caenorhabditis elegans; DISC, death-inducing signaling complex; CARD, caspaserecruitment domain; cryo-EM, electron cryo-microscopy; (d)ADP, (deoxy)adenosine diphosphate; Dark, Drosophila Apaf-1 related killer; DD, death domain; DED, death effector domain; (d)ATP, (deoxy)adenosine triphosphate; FADD, Fas-associated death domain; Hac-1, homolog of Apaf-1 and CED-4; ICE, interleukin 1 $\beta$ converting enzyme; PETCM, alpha-(trichloromethyl)-4-pyridineethanol; PIDD, p53-induced protein with a death domain; TNF, tumor necrosis factor; WT, wild type Received 09.6.06; revised 14.7.06; accepted 14.7.06; Edited by S Kumar; published online 15.9.06
} 


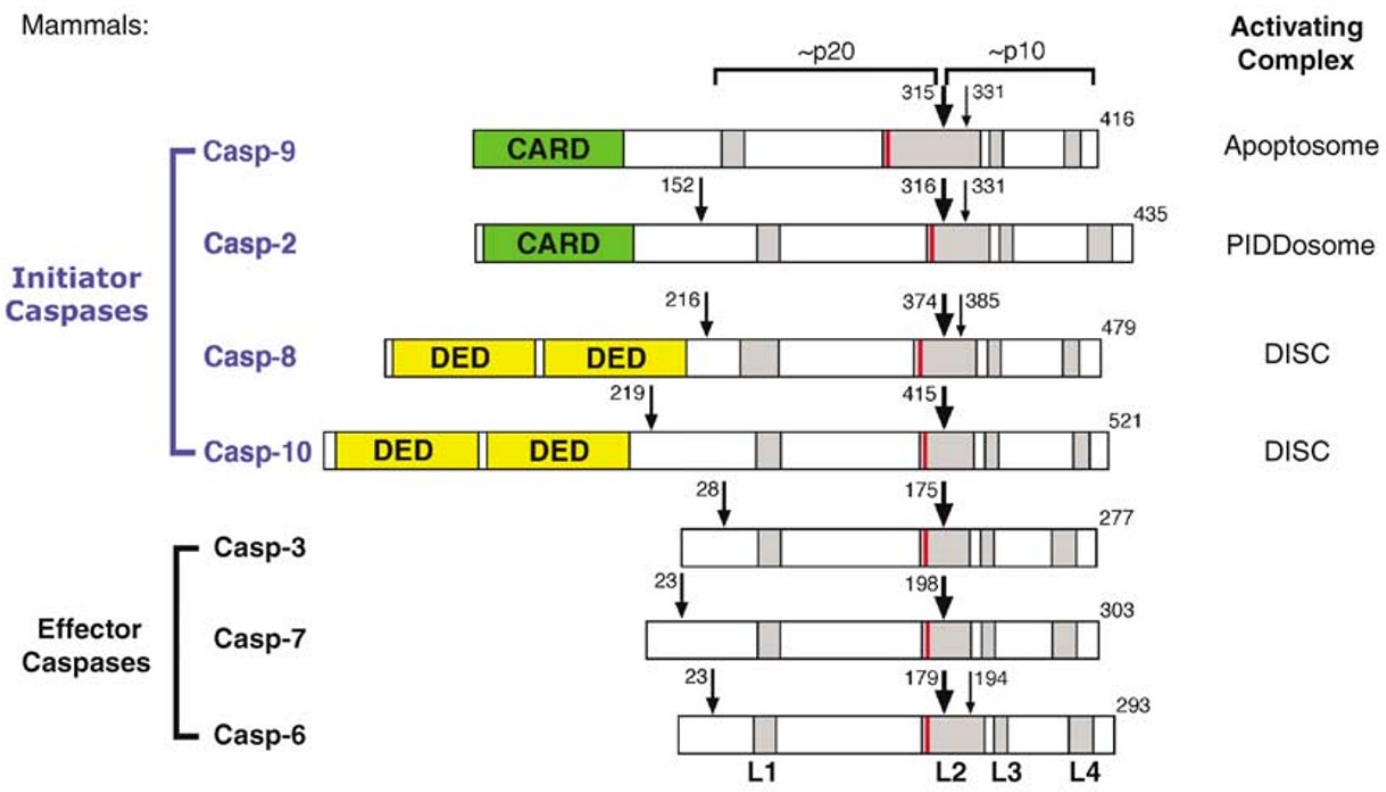

Drosophila:

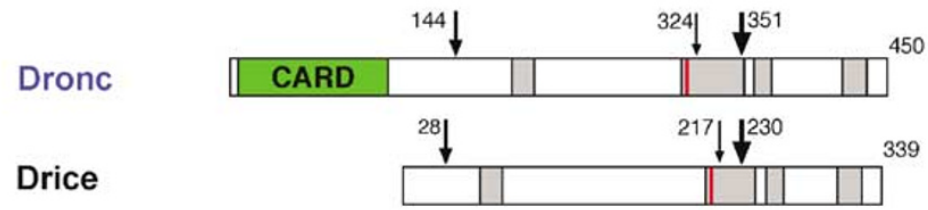

Dark/Hac-1/Dapaf-1 Apoptosome

C. elegans

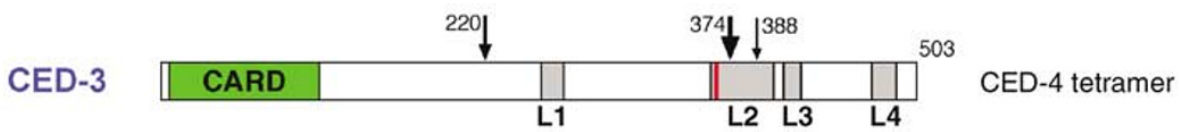

Figure 1 Apoptotic caspases discussed in the review. Six initiator caspases and four effector caspases in three model species are drawn to scale. The position of the first intrachain cleavage (between the large and small subunits) is highlighted by a large arrow whereas additional cleavages are represented by medium and small arrows. The prodomains in initiator caspases invariably contain homotypic interaction motifs, such as the CARD and the DED. ${ }^{33}$ The four surface loops, labeled L1 through L4, form the active site of a caspase. ${ }^{7}$ The catalytic cysteine residue is shown as a red line at the beginning of loop L2. The p20 and p10 subunits together form a caspase monomer

for the activation of some initiator caspases, it has only modest effect on the catalytic activity of other initiator caspases and may not be required for their activation. ${ }^{10-12}$ For example, the fully processed caspase- 9 in isolation exhibits a low level of catalytic activity, similar to that of the unprocessed caspase-9 zymogen.

How is an initiator caspase activated then? The answer lies on a specific adaptor protein complex. The activation of caspase-2, caspase-8, and caspase- 9 in mammalian cells depends on the PIDDosome,$^{13}$ the death-inducing signaling complex (DISC), ${ }^{14}$ and the apoptosome, ${ }^{15-19}$ respectively (Figure 2). The primary component of the apoptosome is the apoptotic protease-activating factor 1 (Apaf-1). In the fruit fly Drosophila, the activation of Dronc (homolog of caspase-9) requires an octameric protein complex involving Drosophila Apaf-1 related killer (Dark) ${ }^{20}$ (also known as Hac-1 (homolog of Apaf- 1 and CED-4) ${ }^{21}$ or Dapaf-1, ${ }^{22}$ a homolog of Apaf-1). In the nematode $C$. elegans, the activation of CED-3 caspase zymogen is facilitated by CED- $4,{ }^{23}$ which exhibits significant sequence homology to Apaf-1. In each case, the initiator caspase is recruited into and activated within a specific adaptor protein complex. These adaptor protein complexes are always oligomeric and generally referred to as apoptosomes.

The assembly of an apoptosome is a highly regulated process. Recent biochemical and structural investigation has revealed significant insights into the assembly and function of apoptosomes. In this review, we describe our current knowledge on this topic and focus on the latest development.

\section{PIDDosome for Caspase-2 Activation}

Caspase-2 is the second caspase identified in mammalian cells and is required in stress-induced apoptosis. ${ }^{24}$ It contains all hallmarks of an initiator caspase, including a caspaserecruitment domain (CARD) at its amino-terminus. The crystal structure of the CARD-deleted, activated caspase-2 revealed an unanticipated disulfide bond at the dimeric interface, ${ }^{25}$ 


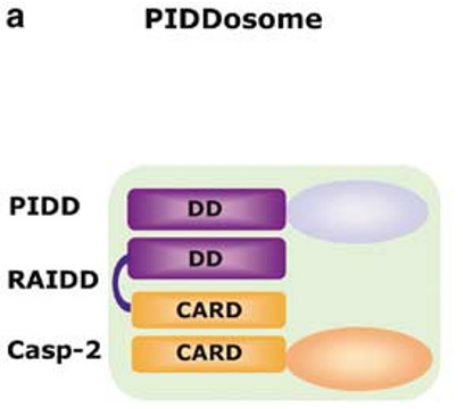

d Dark-apoptosome

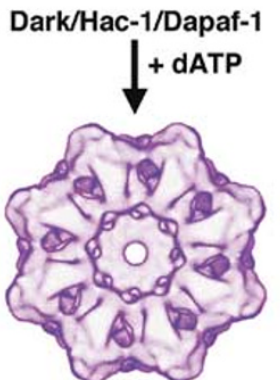

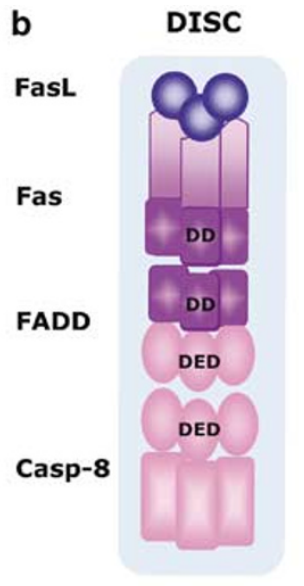

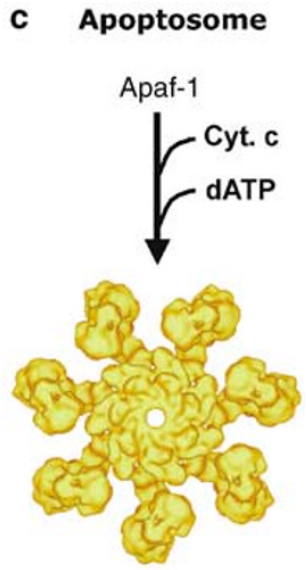

e

CED-4-tetramer

$($ CED-4) 2 :CED-9

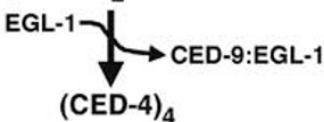

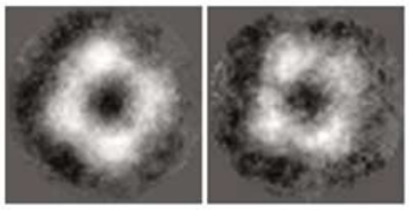

Figure 2 Adaptor protein complexes, or apoptosomes, that are responsible for the activation of initiator caspases. The activation of caspase-2, caspase-8, and caspase-9 in mammalian cells are mediated by the PIDDosome (a),${ }^{13}$ the DISC (b), ${ }^{14}$ and the apoptosome $(\mathbf{c}),{ }^{15-19}$ respectively. PIDDosome contains at least three components, PIDD, RAIDD, and caspase-2. ${ }^{13}$ DISC is assembled following binding of death ligand to its receptor and contains FADD and caspase-8 (or -10). Apoptosome is composed of seven molecules of Apaf- 1 bound to cytochrome $c$ in the presence of ATP/dATP. Activation of Dronc in Drosophila and CED-3 in C. elegans is mediated by the octameric Darkapoptosome (d) and CED-4 tetramer (e), respectively. Images of the various apoptosomes were adapted from previous publications ${ }^{55,59,65}$

suggesting a critical role for dimerization. Subsequent characterization of caspase-2 activation in vitro showed that the autocatalytic intrachain cleavage significantly stabilizes dimer formation and drastically enhances the catalytic activity of caspase-2. ${ }^{26}$ However, the disulfide bond observed in the crystal structure appears to be dispensable for caspase-2 dimerization, as mutation of the corresponding Cys residue at the dimeric interface did not affect the ability of recombinant caspase-2 to dimerize or to undergo autocatalytic cleavage. ${ }^{26}$

The activation of caspase- 2 in cells requires the assembly of a large protein complex with a molecular weight in excess of $670 \mathrm{kDa}^{13,27}$ Characterization of this complex revealed the presence of an adaptor protein RAIDD and the p53-induced protein with a death domain (PIDD). ${ }^{13}$ Subsequently, the caspase-2-activating complex has been referred to as the PIDDosome (Figure 2a). ${ }^{13}$ In addition to the activated caspase-2, structural information on the PIDDosome is available on the isolated CARD domain of RAIDD, ${ }^{28}$ which is required for binding to the CARD domain of caspase-2, and the isolated death domain (DD) of RAIDD, ${ }^{29}$ which is thought to interact with the DD of PIDD. At present, the stoichiometry among PIDD, RAIDD, and caspase- 2 remains enigmatic. It is also unclear whether any additional cofactor is involved in the assembly of PIDDosome. Most importantly, the molecular mechanism by which the PIDDosome facilitates the activation of caspase-2 remains unknown. Does PIDDosome simply promote the autocatalytic cleavage of caspase-2 zymogen? Or can PIDDosome allosterically regulate caspase-2 activity in a way similar to the case of apoptosome to caspase-9 (see later)? The answer to the latter question is likely to be negative, because, in contrast to caspase-9, caspase-2 contains a caspase cleavage site between the caspase unit and the amino-terminal CARD that is thought to be responsible for the recruitment of caspase-2 into the PIDDosome (Figure 1). Nonetheless, definitive answers to these questions await experimental evidence.

\section{DISC for Caspase-8 Activation}

The extrinsic apoptosis pathway is triggered by the binding of a specific death ligand, such as FasL (also known as CD95L), to a specific transmembrane death receptor, such as Fas (also known as APO-1/CD95). ${ }^{2}$ The death receptors belong to the tumor necrosis factor (TNF) family, which contains a single DD in the intracellular compartment. Interestingly, the discovery of the DISC preceded and contributed to the identification of caspase-8. A large protein complex involving the activated Fas death receptor was found to assemble in the 
dying cells and named DISC. ${ }^{14}$ One critical component of DISC was identified to be Fas-associated death domain (FADD, also known as MORT1) ${ }^{14,30}$ Another critical component of DISC was identified to be caspase-8 (also known as FLICE/MACH). ${ }^{31,32}$ Although DISC may contain additional cofactors and regulatory proteins (such as FLIPS), Fas, FADD, and caspase- 8 are the three essential and indispensable components (Figure $2 b)^{2}$

The function of DISC is to activate the initiator caspase, caspase-8, and perhaps caspase- 10 as well. ${ }^{2}$ The activated death ligands are homo-trimeric and thus induce oligomerization of the death receptors upon binding. ${ }^{2,33}$ The receptorassociated adapter protein FADD contains a death effector domain (DED) at its amino-terminus and a DD at its carboxyterminus; whereas caspase- 8 has two copies of DED at its amino-terminus. FADD is thought to bind Fas and the procaspase-8 zymogen through homotypic interactions, thus bringing at least three molecules of procaspase-8 into close proximity of one another and facilitating their autoactivation. The protein components of DISC are technically difficult to deal with; consequently, the activation of caspase- 8 by DISC has yet to be reconstituted in vitro using recombinant proteins. Nonetheless, structures of the Fas DD, ${ }^{34}$ FADD DD, ${ }^{35,36}$ and FADD $D^{37}$ have greatly facilitated identification of the interaction interfaces among components of the DISC. ${ }^{38,39}$ More recently, structures of an intact FADD ${ }^{40}$ and the viral FLIP MC159 11,42 and biochemical characterization of the DISC components ${ }^{39,40,42,43}$ allowed proposition of interesting yet speculative models on DISC assembly. Interestingly, the DED and DD of FADD appear to adopt a defined relative orientation, ${ }^{40}$ and the two tandem copies of DED in MC159 are rigidly held together by hydrophobic interactions. ${ }^{41,42}$ These observations serve as important constraints for the modeling of DISC assembly.

At present, our understanding on the molecular mechanism of caspase-8 activation is derived almost entirely from studies on the isolated caspase-8. A prodomain-deleted uncleavable caspase- 8 was found to exist mainly as a monomer by gel filtration; yet the catalytic activity predominantly associated with the fractions that corresponded to dimers. ${ }^{44,45}$ In contrast, the wild-type (WT) caspase-8 exists in an equilibrium between monomers and dimers, with a dissociation constant of approximately $50 \mu \mathrm{M}$, and binding to inhibitors results in enhanced dimerization for the wild type (WT) caspase-8. ${ }^{44}$ These findings strongly argue that dimerization is a crucial factor for caspase- 8 activation and suggest that DISC may facilitate the activation of caspase-8 through dimerization. This conclusion is further supported by the report that $c-F L I P_{L}$ may activate caspase- 8 through hetero-dimerization. ${ }^{46-48}$

\section{Apoptosome for Caspase-9 Activation}

Caspase- 9 is the most thoroughly characterized initiator caspase and its activation is mediated by the apoptosome, a multimeric complex involving Apaf-1, cytochrome $c$, and the cofactor dATP/ATP. Caspase-9 is the only initiator caspase whose activation by the adaptor complex has been successfully reconstituted in vitro using homogeneous recombinant proteins.
Cytochrome $c$, a crucial molecule for energy production in mitochondria, was identified to be an important cofactor for the activation of caspase-3. ${ }^{49}$ Subsequently, the cellular receptor for cytochrome $c$ was identified to be a novel protein, named Apaf- $1 .{ }^{50}$ Cytochrome $c$ and dATP were found to be essential cofactors for the formation of an Apaf-1/caspase- 9 complex, ${ }^{51}$ which subsequently activates the effector caspases, caspase-3 and -7 .

Subsequent characterization revealed that, in the presence of dATP or ATP, cytochrome $c$ and Apaf-1 assembles into an $\sim 1.4 \mathrm{MDa}$ complex, dubbed the 'apoptosome' (Figure 2c). ${ }^{15-19}$ The CARD domain of Apaf-1 in the apoptosome interacts with the prodomain (also a CARD domain) of procaspase- 9 , resulting in the recruitment and subsequent activation of procaspase- 9 zymogen. ${ }^{51,52}$ Surprisingly, caspase- 9 bound to the apoptosome exhibits a catalytic activity that is three orders of magnitude higher than that of the isolated caspase9 , prompting the concept of a holoenzyme. ${ }^{18}$ The primary function of the apoptosome appears to be allosteric regulation of the catalytic activity of caspase-9. Supporting this notion, procaspase- 9 zymogen possesses a basal level of activity in the absence of the activation cleavage ${ }^{10}$ and this activity can be upregulated to the same level as the cleaved caspase- 9 by the apoptosome. ${ }^{11}$

Apaf-1, the central component of the apoptosome, has three distinct domains, an $\mathrm{N}$-terminal CARD, an expanded nucleotide-binding domain, and 12-13 WD40 repeats at its carboxy-terminal half. The CARD is responsible for interactions with the prodomain of caspase-9; this interaction is essential to the recruitment and activation of procaspase9. ${ }^{51,52}$ The CARD and the nucleotide-binding domains are responsible for the oligomerization of Apaf- 1 in the presence of cytochrome $c$ and dATP, whereas the WD-40 repeats are thought to interact with cytochrome $c$ as removal of this domain in Apaf-1 results in constitutive binding and activation of caspase-9. There are at least four distinct Apaf-1 splicing variants, but they all contain these three essential domains.

Apaf- 1 exists in an inactive conformation in cells and is activated through binding to cytochrome $c$ and dATP. ${ }^{51}$ The structure of a WD40-deleted Apaf-1 revealed the underlying mechanism by which Apaf-1 maintains itself in an inactive state prior to dATP/ATP binding and explained why Apaf-1 needs the nucleotide for activation. ${ }^{53}$ In the structure, five distinct domains, CARD, three-layered $\alpha / \beta$ domain, helical domain I, winged-helix domain, and helical domain II, pack closely against each other through extensive interdomain interactions (Figure 3a). ${ }^{53}$ These interactions result in the partial burial of the caspase-9-binding interface. Unexpectedly, the bound nucleotide is ADP, which is deeply buried and serves as an organizing center to strengthen interactions among these four adjoining domains. ${ }^{53}$ Structural analysis suggests that binding of nucleotide may induce significant conformational changes in Apaf-1 and that these conformational changes may drive the formation of the caspase-9activating apoptosome.

How does the activated Apaf-1 assemble into an apoptosome? Structure of the apoptosome, determined by electron cryo-microscopy (cryo-EM), reveals a wheel-shaped complex with seven-fold symmetry (Figure $3 \mathrm{~b}$, left panel). ${ }^{54,55}$ The CARD and the expanded nucleotide-binding domain of Apaf-1 

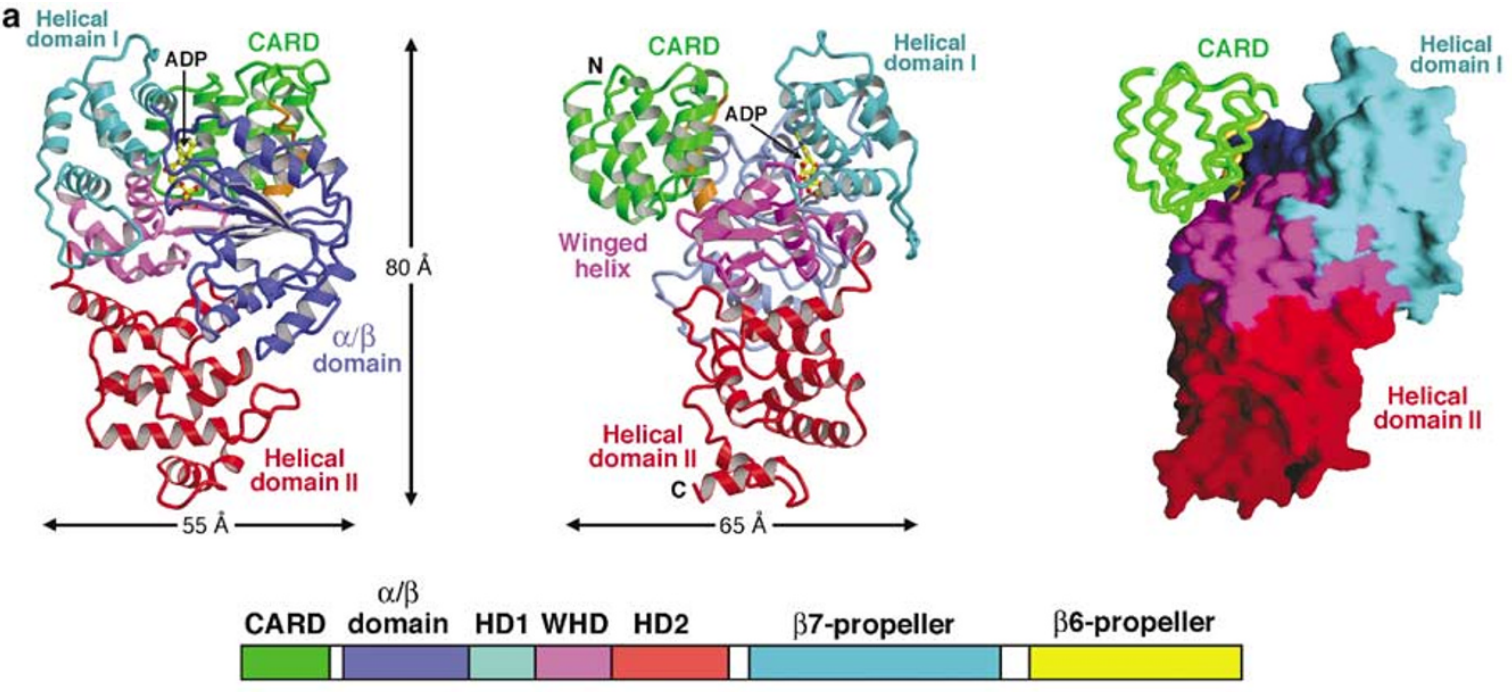

b
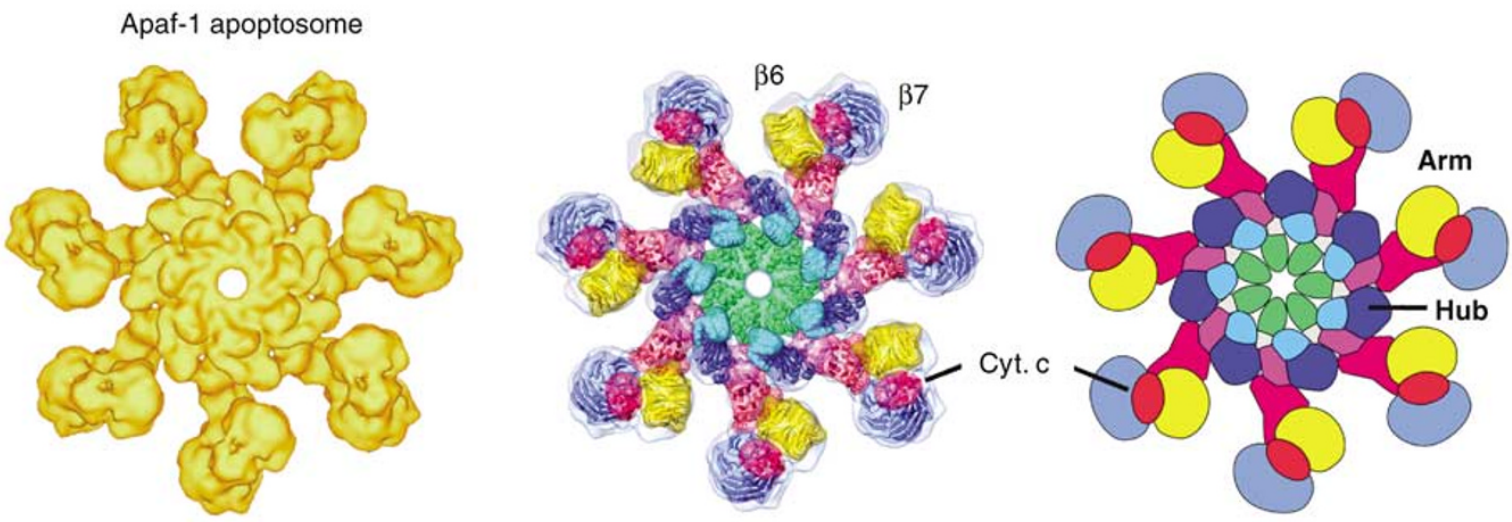

C

Dark apoptosome
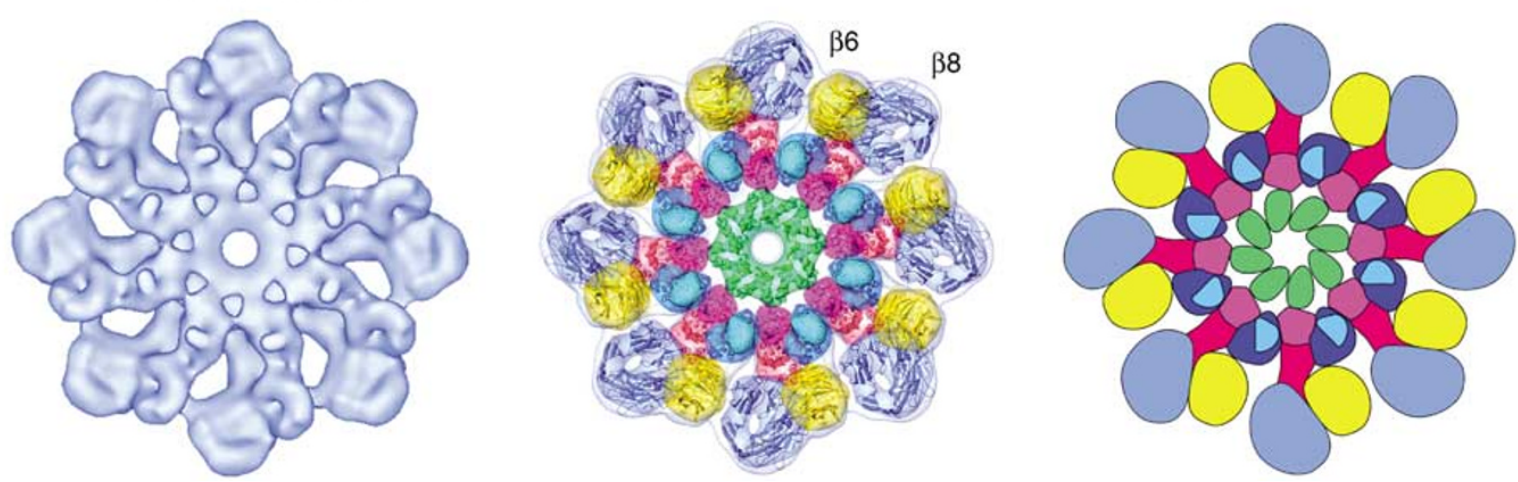

Figure 3 Domain organization of Apaf-1 and Dark apoptosomes. (a) Overall structure of the WD40-deleted Apaf-1. ${ }^{53}$ The left and middle panels display two perpendicular views of the ribbon diagram of the structure of Apaf-1 (residues 1-591, bound to ADP). The WD40-deleted Apaf-1 sequentially comprises five distinct domains, CARD (colored green), an $\alpha / \beta$ domain (blue), helical domain I (HD1, cyan), a winged-helix domain (WHD, magenta), and helical domain II (HD2, red). These five domains pack against one another to generate a relatively compact structure. ADP binds to the hinge region between the $\alpha / \beta$-fold and HD1 but is also coordinated by two critical residues from the WHD. The right panel shows the structure in surface representation except the CARD domain. (b) Domain organization in Apaf-1 apoptosome. ${ }^{55}$ Left panel shows a top view of the apoptosome.$^{55}$ Middle panel shows the proposed domain organization in the apoptosome within semitransparent surfaces ${ }^{55}$ Right panel shows a cartoon model of the apoptosome.$^{55}$ Color-coding scheme for the middle and right panels is the same as in (a). (c) Domain organization in the Dark-apoptosome. ${ }^{59}$ Left panel shows a top view of the Dark-apoptosome. ${ }^{59}$ Middle panel shows the proposed domain organization in the Dark-apoptosome within semitransparent surfaces. ${ }^{59}$ Right panel shows a cartoon model of the Dark-apoptosome ${ }^{59}$ Color-coding scheme for the middle and right panels is the same as in (a). Compared to Apaf-1-apoptosome, the $\alpha / \beta$ domain of Dark shifts outwards within the central hub to contact the $\beta 6$-propeller. Panels $b$ and $c$ were reproduced from the original publication with permission ${ }^{59}$ 
are located at the central hub whereas the WD40 repeats constitute the extended spokes. Knowledge of the crystal structure of the autoinhibited Apaf-1 at an atomic resolution allowed a detailed assignment of the individual domains in the EM map (Figure 3b, middle and right panels). ${ }^{55}$ Despite the relatively low resolution, the cryo-EM structure confirms the structural involvement of cytochrome $c$ in the formation of the apoptosome. Docking of procaspase- 9 to this apoptosome resulted in a dome-shaped structure in the center; however, the bulk of procaspase- 9 was not visible in these cryo-EM studies. ${ }^{54}$ Limited by the low resolution, the apoptosome structure does not allow assignment of atomic interactions. Consequently, the molecular underpinnings of caspase9 activation remain to be elucidated.

The kinetics and regulation of apoptosome assembly are complex and are only beginning to be appreciated. The bound nucleotide in Apaf-1 appears to undergo dynamic changes during apoptosome assembly. Cytochrome $c$ binding to the monomeric Apaf-1 was reported to induce hydrolysis of dATP to dADP, which was subsequently replaced by exogenous dATP. ${ }^{56}$ Hydrolysis of dATP and subsequent exchange of dADP by dATP were thought to be two required steps for apoptosome assembly. ${ }^{56}$ It is conceivable that these two steps can be regulated by other cellular factors, such as nucleotide exchange factors and/or ATPase activating proteins, to impact on apoptosome assembly, although no specific factor has been reported to date. In a recent study, the oncoprotein prothymosin-alpha was shown to negatively regulate caspase- 9 activation by inhibiting apoptosome formation. ${ }^{57} \mathrm{~A}$ small molecule alpha-(trichloromethyl)-4-pyridineethanol (PETCM) relieved prothymosin-alpha inhibition and allowed apoptosome formation, although how PETCM accomplished this task remains unclear. ${ }^{57}$

\section{Dark-Apoptosome for Dronc Activation}

Dronc is the Drosophila orthologue of the mammalian initiator caspase- 9 and is required for programmed cell death during the normal development of fruit flies. One important downstream target of Dronc is the effector caspase Drice, an orthologue of mammalian caspase-3. The activation of Dronc in Drosophila cells requires Dark (also known as Hac-1 or Dapaf-1), ${ }^{20-22}$ the orthologue of mammalian Apaf-1. However, in contrast to caspase-9, the prodomain of Dronc is cleaved off the caspase unit in Drosophila cells, ${ }^{58}$ suggesting a mode of activation different from that for caspase-9.

Dark assembles into an apoptosome in the presence of dATP. ${ }^{59}$ In contrast to the Apaf-1-apoptosome, cytochrome $c$ was not required for assembly of the Dark-apoptosome and when added, cytochrome $c$ did not bind to the Darkapoptosome. $^{59}$ Cryo-EM structure of the Dark-apoptosome revealed two wheel-shaped particles associated face-to-face, each involving eight molecules of Dark (Figure 3c). ${ }^{59}$ Relevant crystal structures were used to model the Dark-apoptosome. Structural analysis showed that, despite some apparent differences, the Dark-apoptosome and the Apaf-1-apoptosome share a number of important features. For example, placement of the CARD domain in the apoptosome is highly conserved. This analysis also suggested that a single wheelshaped particle likely represent the functional Dark-apopto- some in Drosophila. It is important to note that, although Dark and Apaf-1 both belong to the AAA + ATPases, the proposed models of oligomerization for Dark and Apaf-1 are quite different from that of other AAA + ATPases. ${ }^{60}$

Limited insights into Dronc activation came from in vitro biochemical characterization. In contrast to caspase-9, autocatalytic cleavage of Dronc results in a drastic enhancement of its catalytic activity in vitro. ${ }^{61}$ The autocleaved Dronc formed a homo-dimer whereas the uncleaved Dronc zymogen existed exclusively as a monomer. ${ }^{61}$ Thus, the autocatalytic cleavage in Dronc induces its stable dimerization, which presumably allows the two adjacent monomers to mutually stabilize their active sites, leading to activation. Crystal structure of a prodomain-deleted Dronc zymogen revealed an unproductive conformation at the active site, which is consistent with the observation that the zymogen remains catalytically inactive. ${ }^{61}$

\section{CED-4-Apoptosome for CED-3 Activation}

Genetic analysis in $C$. elegans led to the identification of four genes, egl-1, ced-9, ced-4, and ced-3, that control the death of 131 somatic cells during hermaphrodite development. ${ }^{23,62}$ When the cells are programmed to die, the CED-3 zymogen is thought to be activated by the adaptor molecule CED-4. In normal cells, the proapoptotic protein CED-4 is sequestered by the mitochondria-bound protein CED-9, thus unable to activate CED-3. During apoptosis, the inhibitory CED-4/CED9 interaction is disrupted by the proapoptotic protein EGL-1, which is transcriptionally activated in cells destined to die. The released CED-4 is thought to undergo homo-oligomerization, which then facilitates the activation of CED-3. ${ }^{63}$

Recent investigation suggested that CED-9 and CED-4 formed a 2:2 heterotetrameric complex. ${ }^{64}$ However, structural investigation revealed that one molecule of CED-9 interacts with an asymmetric dimer of CED-4 but only specifically recognizes one of the two CED-4 molecules through an extensive interface dominated by hydrogen bonds. ${ }^{65,66}$ This specific interaction prevents CED-4 from activating CED-3. $E G L-1$ binding induces significant conformational changes in CED-9 that result in the dissociation of CED-9 from the CED-4 dimer. ${ }^{65}$ The freed CED-4 dimer further dimerizes to form a tetramer, which facilitates the autoactivation of CED-3. Thus, the CED-4 apoptosome is a tetramer of CED-4. How CED-4 assembles into a tetramer and exactly how the CED-4 apoptosome facilitates CED-3 autoactivation await further investigation.

\section{Models of Initiator Caspase Activation}

Induced proximity model. Although the activation mechanism of effector caspases has been documented at molecular detail, ${ }^{67,68}$ how initiator caspases are activated remains inconclusive. The induced proximity model states that the initiator caspases autoprocess themselves when brought into close proximity of each other. ${ }^{8}$ This model elegantly summarizes experimental observations made by four independent laboratories, led by Dixit and Salvesen, ${ }^{69}$ Yang and Baltimore, ${ }^{63,70}$ Alnemri, ${ }^{71}$ and Spenser. ${ }^{72}$ All four 
laboratories used a similar experimental approach, in which the target caspases were fused with a heterologous dimerization domain such as tandem FK506-binding domains (FKBPs) (Figure 4a). In all cases, the target caspases were processed upon induced oligomerization. Once recruited into the relevant apoptosomes, the initiator caspases are brought into close proximity of one another, followed by autoactivation. In this regard, the induced proximity model faithfully summarizes the process of initiator caspase activation at a general level but does not explain how the initiator caspases are activated at a mechanistic level.

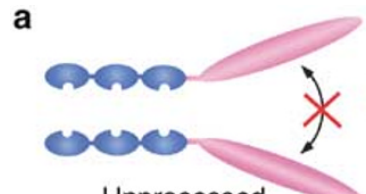

Unprocessed

FKBP : FK1012

b

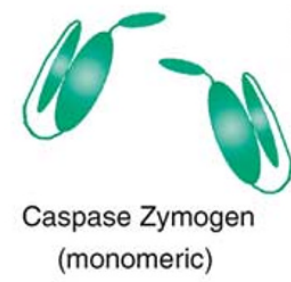
Proximity-driven
Dimerization

Caspase zymogen
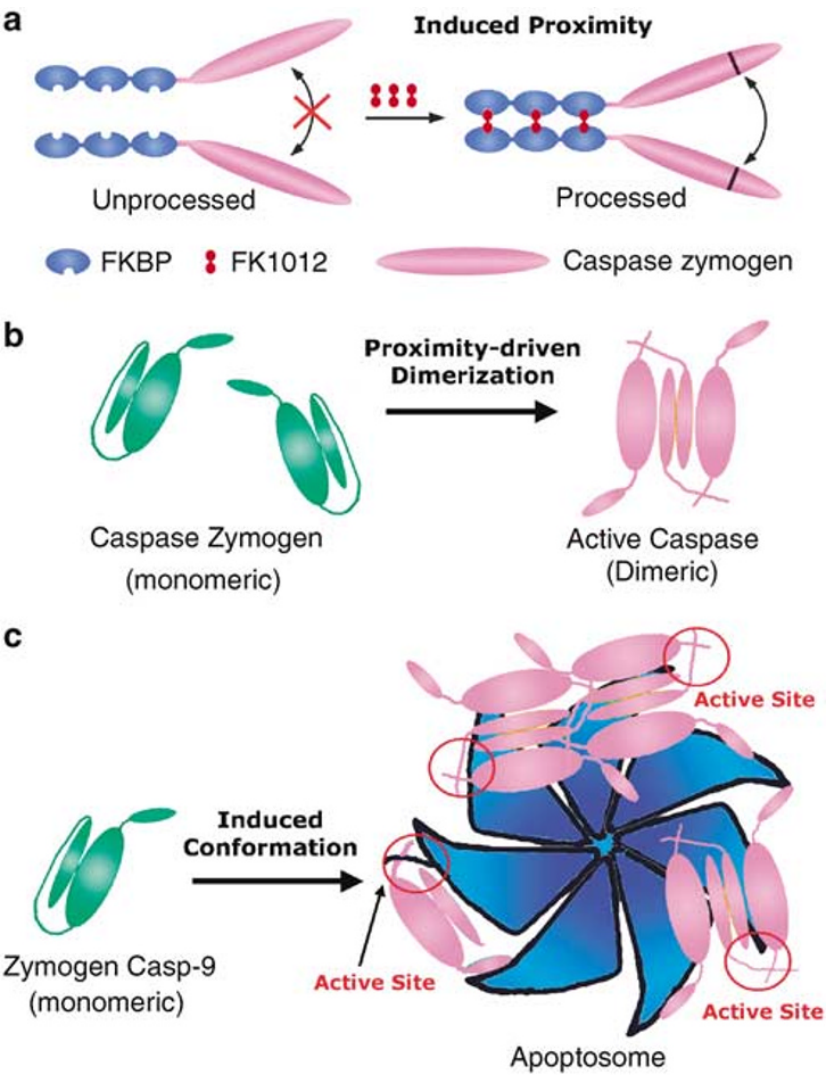

Figure 4 Models of initiator caspase activation. (a) Induced-proximity model. ${ }^{8}$ The induced proximity model states that the initiator caspases auto-process themselves when brought into close proximity of each other. ${ }^{8}$ The primary supporting evidence was obtained using a fusion protein between the target caspases and a heterologous dimerization domain, such as tandem FKBPs shown here. Binding to the dimeric ligand FK1012 brings together the tethered caspases, resulting in their activation. (b) Proximity-driven dimerization model. ${ }^{73}$ This model states that dimer formation drives the activation of initiator caspases and the adaptor protein complexes serve to promote dimerization by increasing the local concentrations of initiator caspases. (c) Induced conformation model. ${ }^{12,80}$ This model states that the activated conformation of the active site for a given initiator caspase is the ultimate reason for its activation and is attained through direct interaction with the adaptor protein complex or through homo-oligomerization facilitated by the adaptor protein complex. Three scenarios are depicted here. One scenario is that caspase-9 is activated as a monomer, with its active site conformation stabilized directly by the apoptosome. The second scenario is that caspase-9 is activated as a homo-dimer, as proposed by the proximity-driven dimerization model,$^{73}$ with its active site conformation stabilized by dimer formation and dimer interactions with the apoptosome. The third scenario is that caspase- 9 is activated as a higher-order homo-oligomer, with its active site conformation stabilized both by the oligomer and the apoptosome
Proximity-driven dimerization model represents a refinement of the Induced Proximity model. ${ }^{73}$ Caspases were generally thought to be homo-dimers. Thus, it came as a surprise that both procaspase- 9 zymogen and the processed caspase- 9 mainly exist as a monomer in solution. ${ }^{74,75}$ Using gel filtration, the processed caspase- 9 monomers were separated from the dimers and were found to be catalytically inactive. $^{74}$ In contrast, the dimer fractions were highly active. ${ }^{74}$ These observations gave rise to the proposal that dimer formation may drive the activation of caspase- 9 (Figure 4b). ${ }^{74}$

Based on the proximity-driven dimerization model, the heptameric Apaf-1-apoptosome can recruit multiple molecules of inactive procaspase- 9 into close proximity of one another. The high local concentrations of procaspase- 9 monomers in the apoptosome was predicted to favor dimerization and hence activation. ${ }^{54,74}$ This model is consistent with the observed second-order activation of caspase9 by a mini-apoptosome. ${ }^{76}$ In addition, a heterologous fusion protein between a dimeric leucine zipper and caspase- 9 resulted in significant enhancement of its catalytic activity, which argues that dimerization of caspase- 9 may be sufficient for its activation. ${ }^{77}$ In contrast to these studies, an engineered caspase-9, which exists as a constitutive homo-dimer in solution, exhibited a much lower level of catalytic activity compared to the apoptosome-activated caspase-9. ${ }^{78}$ These contrasting observations highlight the necessity to conclusively define the mechanism by which apoptosome facilitates the activation of caspase-9, perhaps by elucidating the structure of the apoptosome-caspase- 9 holoenzyme.

The mammalian caspase-2 and caspase-8 and the Drosophila Dronc appear to share a common mechanism of activation. Zymogen of each of the three initiator caspases was found to exist mainly as a monomer by gel filtration. ${ }^{26,44,45,61}$ In contrast, the auto-cleaved WT caspase-8 and Dronc exist in an equilibrium between monomers and dimers, with dissociation constants of approximately 50 and $0.27 \mu \mathrm{M}$, respectively. ${ }^{44,61}$ The processed WT caspase- 2 also preferentially formed homo-dimers. ${ }^{26}$ These findings strongly suggest that dimerization is a crucial factor for the activation of caspase-2, caspase-8, and Dronc, and that the autocatalytic cleavage in these mammalian initiator caspases plays an important role for their activation. These observations also imply that PIDDosome, DISC, and Dark-apoptosome may serve to facilitate the dimerization and auto-catalytic cleavage of caspase-2, caspase-8, and Dronc, respectively. How this is accomplished remains to be investigated.

Induced conformation model. The fact that caspase- 9 exhibits a much higher level of catalytic activity in the apoptosome demonstrates that a conformational change must have occurred in the active site of the apoptosomebound caspase-9. The proximity-driven dimerization model argues that this conformational change is identical to that seen in the monomer-to-dimer transition of an initiator caspase. There are additional possibilities. One model is that the apoptosome may directly activate monomeric caspase- 9 through modification of its active site conformation (Figure 4c). ${ }^{75}$ Under this scenario, one way the apoptosome may activate the monomeric caspase- 9 is to 
bind to its surface that is required for its homo-dimerization thus stabilizing the productive conformation of the active site. Consistent with this model, the BIR3 domain of XIAP specifically hetero-dimerizes with caspase- 9 through an interface that is also required for the homo-dimerization of caspase-9. ${ }^{75}$ This model could also explain the observed phenotype of the interface mutant caspase- $9 .{ }^{45}$

An alternative model is that the apoptosome assembles the dimeric caspase-9 into a higher-order complex (Figure 4c), which results in the modification of the active site conformation for enhanced activity. ${ }^{12}$ Consistent with this hypothesis, in the crystals of the inhibitor-bound caspase- 9 , caspase- 9 exists as a dimer of homo-dimer or a homo-tetramer. ${ }^{74}$ The specific interactions between the two homo-dimers are more extensive than regular crystal packing contacts and directly affect the conformation of the active sites. ${ }^{74} \mathrm{~A}$ third possibility is that the apoptosome simply forces the WT monomeric caspase-9 to dimerize (Figure 4c); but this process must involve not just increased local concentration but also additional interface between the apoptosome and caspase- $9 .^{79}$

These hypotheses are collectively termed 'induced conformation' model (Figure 4c), in which conformational changes at the active site are regarded as the ultimate reason for caspase activation. ${ }^{12}$ Although available data are insufficient to differentiate among various specific models, exquisite conformational changes of caspase- 9 must be induced upon binding to the apoptosome. ${ }^{79}$ Previous biochemical studies revealed that the CARD domain of Apaf-1 oligomerizes caspase- 9 into a multimeric complex, with a molecular weight of approximately $300 \mathrm{kDa}^{79}$ Because Apaf-1 CARD only forms a stable hetero-dimer with the prodomain of caspase$9,51,52$ the formation of this large 'CARD/caspase- 9 ' complex must involve an additional interface between the catalytic subunit of caspase-9 and Apaf-1 CARD. This complex was thought to represent the strapped-down version of the apoptosome as caspase- 9 within this complex exhibited significantly elevated catalytic activity compared to the isolated caspase- $9 .{ }^{79}$ Regardless of the catalytic activity, the formation of the $300 \mathrm{kDa}$ complex between Apaf-1 CARD and caspase- 9 demonstrates that other interactions, in addition to those between their respective CARD domains, must exist between caspase- 9 and Apaf-1. This interaction is predicted to play an important role in the activation of caspae- 9 within the apoptosome.

\section{Conclusion and Perspective}

Our understanding on apoptosome assembly is far from complete. On the one hand, Apaf-1-apoptosome, Darkapoptosome, and CED-4-apoptosome have been assembled in vitro using homogeneous recombinant proteins. But kinetics and regulation of apoptosome assembly are only beginning to be investigated. The differential role of ATP/ dATP in the assembly of various apoptosomes is yet to be examined. On the other hand, DISC and PIDDosome have yet to be biochemically characterized. The in vitro reconstitution of a specific apoptosome is the first essential step in elucidation of the activation mechanism for the corresponding initiator caspase.
The only known function of the apoptosome is to facilitate the activation of initiator caspases. However, despite intense investigation, we have only studied the mechanism of activation for a few initiator caspases, mostly relying on isolated proteins without the involvement of the relevant apoptosomes. The induced proximity model summarizes the general process of initiator caspase activation. ${ }^{8}$ The proximity-driven dimerization model describes how initiator caspases respond to induced proximity and offers a specific explanation to how the initiator caspases are activated. ${ }^{73}$ No matter how caspases are activated, enhanced catalytic activity must be correlated with altered active site conformation. In this regard, the induced conformation model was proposed to explain the activation of initiator caspase activation. ${ }^{12,80}$ Both proximity-driven dimerization and induced conformation hypotheses can be viewed as extensions of the induced proximity model.

The induced conformation model is different from the proximity-driven dimerization model in that different aspects are emphasized for the activation of initiator caspases - the former stresses the importance of active site conformation whereas the latter highlights the dimerization process. The induced conformation model states that the activated conformation of the active site for a given initiator caspase is attained through direct interaction with the relevant apoptosome or through homo-oligomerization facilitated by the relevant apoptosome. These two models are not always mutually exclusive. For example, the essence of proximitydriven dimerization for an initiator caspase is to orient the active site conformation for more efficient substrate binding and catalysis. ${ }^{9}$ For some initiator caspase(s), dimerization might be sufficient for inducing the correct conformation that is needed for its activation. In this case, these two models are in agreement with each other.

The effector caspase zymogens are constitutively dimeric in solution; but their active sites exist in an inhibited conformation. ${ }^{67,68}$ The intrachain cleavage, mediated by a specific initiator caspase, allows a critical loop in one caspase monomer to change conformation and to stabilize the active site loops in the adjacent monomer. ${ }^{67,68}$ In this regard, both effector and initiator caspases share the same basic mechanism for their catalytic activation - attaining an activated conformation at the active site, although the means by which they achieve this conformation are different. While effector caspases only require a specific intrachain cleavage, the initiator caspases rely on adaptor protein complexes - the apoptosomes. The underlying molecular mechanisms by which the apoptosome complexes activate initiator caspases remain to be elucidated by biochemistry, biophysics, and structural biology.

Acknowledgements. We apologize to those colleagues whose papers were not cited due to a strict reference limit. We would like to thank C Akey and $\mathrm{N}$ Yan for help with figure preparation and members of the Shi laboratory for discussion. This work is supported by the NIH.

\footnotetext{
1. Danial NN, Korsmeyer SJ. Cell death: critical control points. Cell 2004; 116: 205-219.

2. Peter ME, Krammer PH. The CD95(APO-1/Fas) DISC and beyond. Cell Death Differ 2003; 10: $26-35$.
} 
3. Wang X. The expanding role of mitochondria in apoptosis. Genes Dev 2001; 15 2922-2933.

4. Riedl SJ, Shi Y. Molecular mechanisms of caspase regulation during apoptosis. Nat Rev $\mathrm{Mol}$ Cell Biol 2004; 5: 897-907.

5. Yuan J, Shaham S, Ledoux S, Ellis HM, Horvitz HR. The $C$. elegans cell death gene Ced-3 encodes a protein similar to mammalian interleukin-1 beta-converting enzyme. Cell 1993; 75: $641-652$.

6. Xue D, Shaham S, Horvitz HR. The Caenorhabditis elegans cell-death protein CED-3 is a cysteine protease with substrate specificities similar to those of the human CPP32 protease. Genes Dev 1996; 10: 1073-1083.

7. Shi Y. Mechanisms of caspase inhibition and activation during apoptosis. Mol Cell 2002; 9 459-470.

8. Salvesen GS, Dixit VM. Caspase activation: the induced-proximity model. Proc Natl Acad Sci USA 1999; 96: 10964-10967.

9. Fuentes-Prior P, Salvesen GS. The protein structures that shape caspase activity, specificity, activation and inhibition. Biochem J 2004; 384: 201-232.

10. Stennicke HR, Deveraux QL, Humke EW, Reed JC, Dixit VM, Salvesen GS. Caspase-9 can be activated without proteolytic processing. J Biol Chem 1999; 274: 8359-8362.

11. Srinivasula SM, Saleh A, Hedge R, Datta P, Shiozaki E, Chai J et al. A conserved XIAPinteraction motif in caspase-9 and Smac/DIABLO mediates opposing effects on caspase activity and apoptosis. Nature 2001; 409: 112-116.

12. Shi Y. Caspase activation: revisiting the induced proximity model. Cell 2004; 117: 855-858.

13. Tinel A, Tschopp J. The PIDDosome, a protein complex implicated in activation of caspase2 in response to genotoxic stress. Science 2004; 304: 843-846.

14. Kischkel FC, Hellbardt S, Behrmann I, Germer M, Pawlita M, Krammer PH et al. Cytotoxicity-dependent APO-1 (Fas/CD95)-associated proteins form a death-inducing signaling complex (DISC) with the receptor. EMBO J 1995; 14: 5579-5588.

15. Zou H, Li Y, Liu X, Wang X. An APAF-1-cytochrome $c$ multimeric complex is a functional apoptosome that activates procaspase-9. J Biol Chem 1999; 274: 11549-11556.

16. Saleh A, Srinivasula SM, Acharya S, Fishel R, Alnemri ES. Cytochrome $c$ and dATPmediated oligomerization of Apaf-1 is a prerequisite for procaspase-9 activation. $J$ Biol Chem 1999; 274: 17941-17945.

17. Hu $Y$, Benedict MA, Ding L, Nunez G. Role of cytochrome $c$ and dATP/ATP hydrolysis in Apaf-1-mediated caspase-9 activation and apoptosis. EMBO J 1999; 18: 3586-3595.

18. Rodriguez J, Lazebnik Y. Caspase-9 and Apaf-1 form an active holoenzyme. Genes Dev 1999; 13: 3179-3184.

19. Cain K, Brown DG, Langlais $C$, Cohen GM. caspase activation involves the formation of the aposome, a large $(\sim 700 \mathrm{kDa})$ caspase-activating complex. J Biol Chem 1999; 274 22686-22692

20. Rodriguez A, Oliver H, Zou H, Chen $\mathrm{P}$, Wang X, Abrams JM. Dark is a drosophila homolog of Apaf-1/CED-4 and functions in an evolutionarily conserved death pathway. Nat Cell Biol 1999; 1: 272-279.

21. Zhou L, Song Z, Tittel J, Steller H. HAC-1, a Drosophila homolog of Apaf-1 and CED-4 functions in developmental and radiation-induced apoptosis. Mol Cell 1999; 4: 745-755.

22. Kanuka H, Sawamoto K, Inohara N, Matsuno K, Okano H, Miura M. Control of the cell death pathway by Dapaf-1, a Drosophila Apaf-1/CED-4-related caspase activator. Mol Cell 1999; 4: 757-769.

23. Lettre G, Hengartner MO. Developmental apoptosis in C. elegans: a complex CEDnario. Nat Rev Mol Cell Biol 2006; 7: 97-108.

24. Lassus $P$, Opitz-Araya X, Lazebnik $Y$. Requirement for caspase-2 in stress-induced apoptosis before mitochondrial permeabilization. Science 2002; 297: 1352-1354.

25. Schweizer A, Briand C, Grutter MG. Crystal structure of caspase-2, apical initiator of the intrinsic apoptotic pathway. J Biol Chem 2003; 278: 42441-42447.

26. Baliga BC, Read SH, Kumar S. The biochemical mechanism of caspase-2 activation. Cell Death Differ 2004; 11: 1234-1241.

27. Read SH, Baliga BC, Ekert PG, Vaux DL, Kumar S. A novel Apaf-1-independent putative caspase-2 activation complex. J Cell Biol 2002; 159: 739-745.

28. Chou JJ, Matsuo H, Duan H, Wagner G. Solution structure of the RAIDD CARD and Mode for CARD/CARD interaction in Caspase-2 and Caspase-9 recruitment. Cell 1998; 94: 171180.

29. Park HH, Wu H. Crystal structure of RAIDD death domain implicates potential mechanism of PIDDosome assembly. J Mol Biol 2006; 357: 358-364

30. Chinnaiyan AM, Tepper CG, Seldin MF, O'Rourke K, Kischkel FC, Hellbardt S et al. FADD MORT1 is a common mediator of CD95 (Fas/APO-1) and tumor necrosis factor receptorinduced apoptosis. J Biol Chem 1996; 271: 4961-4965.

31. Muzio M, Chinnaiyan AM, Kischkel FC, O'Rourke K, Shevchenko A, Ni J et al. FLICE, novel FADD-homologous ICE/CED-3-like protease, is recruited to the CD95 (Fas/APO-1) death - inducing signaling complex. Cell 1996; 85: 817-827.

32. Boldin MP, Goncharov TM, Goltsev YV, Wallach D. Involvement of MACH, a novel MORT1/FADD-interacting protease, in Fas/APO-1- and TNF receptor-induced cell death. Cell 1996; 85: 803-815.

33. Yan N, Shi Y. Mechanisms of apoptosis through structural biology. Annu Rev Cell Dev Bio 2005; $21: 35-56$

34. Huang B, Eberstadt M, Olejniczak ET, Meadows RP, Fesik SW. NMR structure and mutagenesis of the Fas (APO-1/CD95) death domain. Nature 1996; 384: 638-641.
35. Berglund H, Olerenshaw D, Sankar A, Federwisch M, McDonald NQ, Driscoll PC. The three-dimensional solution structure and dynamic properties of the human FADD death domain. J Mol Biol 2000; 302: 171-188.

36. Jeong EJ, Bang S, Lee TH, Park YI, Sim WS, Kim KS. The solution structure of FADD death domain. Structural basis of death domain interactions of Fas and FADD. J Biol Chem 1999; 274: 16337-16342.

37. Eberstadt M, Huang B, Chen Z, Meadows RP, Ng S-C, Zheng L et al. NMR structure and mutagenesis of the FADD (Mort1) death-effector domain. Nature 1998; 392: 941-945.

38. Sandu C, Gavathiotis E, Huang T, Wegorzewska I, Werner MH. A mechanism for death receptor discrimination by death adaptors. J Biol Chem 2005; 280: 31974-31980.

39. Hill JM, Morisawa G, Kim T, Huang T, Wei Y, Wei Y et al. Identification of an expanded binding surface on the FADD death domain responsible for interaction with CD95/Fas. J Biol Chem 2004; 279: 1474-1481.

40. Carrington PE, Sandu C, Wei Y, Hill JM, Morisawa G, Huang T et al. The structure of FADD and its mode of interaction with procaspase-8. Mol Cell 2006; 22: 599-610.

41. Li FY, Jeffrey PD, Yu JW, Shi Y. Crystal structure of a viral FLIP: insights into FLIPmediated inhibition of death receptor signaling. J Biol Chem 2005; 281: 2960-2968.

42. Yang JK, Wang L, Zheng L, Wan F, Ahmed M, Lenardo MJ et al. Crystal structure of MC159 reveals molecular mechanism of DISC assembly and FLIP inhibition. $\mathrm{Mol} \mathrm{Cell}$ 2005; 20: 939-949.

43. Sandu C, Morisawa G, Wegorzewska I, Huang T, Arechiga AF, Hill JM et al. FADD selfassociation is required for stable interaction with an activated death receptor. Cell Death Differ 20062006 May 19 (Epub ahead of print)

44. Donepudi M, Mac Sweeney A, Briand C, Grutter MG. Insights into the regulatory mechanism for caspase-8 activation. Mol Cell 2003; 11: 543-549.

45. Boatright KM, Renatus M, Scott FL, Sperandio S, Shin H, Pedersen IM et al. A unified model for apical caspase activation. Mol Cell 2003; 11: 529-541.

46. Chang DW, Xing Z, Pan Y, Algeciras-Schimnich A, Barnhart BC, Yaish-Ohad S et al. cFLIP $(L)$ is a dual function regulator for caspase-8 activation and CD95-mediated apoptosis. EMBO J 2002; 21: 3704-3714.

47. Micheau O, Thome M, Schneider P, Holler N, Tschopp J, Nicholson DW et al. The long form of FLIP is an activator of caspase-8 at the Fas death-inducing signaling complex. $J$ Biol Chem 2002; 277: 45162-45171.

48. Boatright KM, Deis C, Denault JB, Sutherlin DP, Salvesen GS. Activation of caspases-8 and -10 by FLIP(L). Biochem J 2004; 382: 651-657.

49. Liu X, Kim CN, Yang J, Jemmerson R, Wang X. Induction of apoptosis program in cell-free extracts: requirement for dATP and cytochrome c. Cell 1996; 86: 147-157.

50. Zou H, Henzel WJ, Liu X, Lutschg A, Wang X. Apaf-1, a human protein homologous to $C$. elegans CED-4, participates in cytochrome c-dependent activation of caspase-3. Cell 1997; 90: 405-413.

51. Li P, Nijhawan D, Budihardjo I, Srinivasula SM, Ahmad M, Alnemri ES et al. Cytochrome c and dATP-Dependent Formation of Apaf-1/Caspase-9 Complex Initiates an Apoptotic Protease Cascade. Cell 1997; 91: 479-489.

52. Qin H, Srinivasula SM, Wu G, Fernandes-Alnemri T, Alnemri ES, Shi Y. Structural basis of procaspase-9 recruitment by the apoptotic protease-activating factor 1. Nature 1999; 399 547-555.

53. Riedl SJ, Li W, Chao Y, Schwarzenbacher R, Shi Y. Structure of the apoptotic protease activating factor 1 bound to ADP. Nature 2005; 434: 926-933.

54. Acehan D, Jiang X, Morgan DG, Heuser JE, Wang X, Akey CW. Three-dimensional structure of the apoptosome: implications for assembly, procaspase-9 binding and activation. Mol Cell 2002; 9: 423-432.

55. Yu X, Acehan D, Menetret JF, Booth CR, Ludtke SJ, Riedl SJ et al. A structure of the human apoptosome at $12.8 \mathrm{~A}$ resolution provides insights into this cell death platform. Structure 2005; 13: 1725-1735.

56. Kim HE, Du F, Fang M, Wang X. Formation of apoptosome is initiated by cytochrome cinduced dATP hydrolysis and subsequent nucleotide exchange on Apaf-1. Proc Natl Acad Sci USA 2005; 102: 17545-17550.

57. Jiang X, Kim HE, Shu H, Zhao $Y$, Zhang $H$, Kofron $J$ et al. Distinctive roles of PHAP proteins and prothymosin-alpha in a death regulatory pathway. Science 2003; 299 223-226.

58. Muro I, Monser K, Clem RJ. Mechanism of Dronc activation in Drosophila cells. J Cell Sci 2004; 117: 5035-5041.

59. Yu X, Wang L, Acehan D, Wang X, Akey C. Three-dimensional structure of a double apoptosome formed by the Drosophila Apaf-1 related killer. J Mol Biol 2006; 355: 577-589.

60. Diemand AV, Lupas AN Modeling AAAt ring complexes from monomeric structures. J Struct Biol 20062006 May 7 (Epub ahead of print).

61. Yan N, Huh JR, Schirf V, Demeler B, Hay BA, Shi Y. Structure and activation mechanism of the Drosophila initiator caspase Dronc. J Biol Chem 2006; 281: 8667-8674.

62. Horvitz HR. Worms, life, and death (Nobel Lecture). Chembiochem 2003; 4: 697-711

63. Yang X, Chang HY, Baltimore D. Essential Role of CED-4 oligomerization in CED-3 activation and apoptosis. Science 1998; 281: 1355-1357.

64. Fairlie W, Perugini M, Kvansakul M, Chen L, Huang D, Colman P. CED-4 forms a 2:2 heterotetrameric complex with CED-9 until specifically displaced by EGL-1 or CED-13. Cell Death Differ 2005; 13: 426-434.

65. Yan N, Chai J, Lee ES, Gu L, Liu Q, He J et al. Structure of the CED-4-CED-9 complex provides insights into programmed cell death in Caenorhabditis elegans. Nature 2005; 437 : 831-837. 
66. Yan N, Xu Y, Shi Y. 2:1 Stoichiometry of the CED-4-CED-9 complex and the tetrameric CED-4: insights into the regulation of CED-3 activation. Cell Cycle 2006; $5: 31-34$.

67. Chai J, Wu Q, Shiozaki E, Srinivasula SM, Alnemri ES, Shi Y. Crystal structure of a procaspase-7 zymogen: mechanisms of activation and substrate binding. Cell 2001; 107 399-407.

68. Riedl SJ, Fuentes-Prior P, Renatus M, Kairies N, Krapp S, Huber R et al. Structura basis for the activation of human procaspase-7. Proc Natl Acad Sci USA 2001; 98 14790-14795.

69. Muzio M, Stockwell BR, Stennicke HR, Salvesen GS, Dixit VM. An induced proximity mode for caspase-8 activation. J Biol Chem 1998; 273: 2926-2930.

70. Yang X, Chang HY, Baltimore D. Autoproteolytic activation of pro-caspases by oligomerization. Mol Cell 1998; 1: 319-325.

71. Srinivasula SM, Ahmad M, Fernandes-Alnemri T, Alnemri ES. Autoactivation of procaspase-9 by Apaf-1-mediated oligomerization. Mol Cell 1998; 1: 949-957.

72. MacCorkle RA, Freeman KW, Spencer DM. Synthetic activation of caspases: artificial death switches. Proc Natl Acad Sci USA 1998; 95: 3655-3660.

73. Boatright KM, Salvesen GS. Mechanisms of caspase activation. Curr Opin Cell Biol 2003; 15: 725-731.
74. Renatus M, Stennicke HR, Scott FL, Liddington RC, Salvesen GS. Dimer formation drives the activation of the cell death protease caspase 9. Proc Natl Acad Sci USA 2001; 98: 14250-14255.

75. Shiozaki EN, Chai J, Rigotti DJ, Riedl SJ, Li P, Srinivasula SM et al. Mechanism of XIAP-mediated inhibition of caspase-9. Mol Cell 2003; 11: 519-527.

76. Pop C, Timmer J, Sperandio S, Salvesen GS. The apoptosome activates caspase-9 by dimerization. Mol Cell 2006; 22: 269-275.

77. Yin $Q$, Park HH, Chung JY, Lin SC, Lo YC, da Graca LS et al. Caspase-9 holoenzyme is a specific and optimal procaspase-3 processing machine. Mol Cell 2006; 22: 259-268.

78. Chao Y, Shiozaki EN, Srinivasula SM, Rigotti DJ, Fairman R, Shi Y. Engineering a dimeric caspase-9: a re-evaluation of the induced proximity model for caspase activation. PLoS Biol 2005; 3: e183.

79. Shiozaki E, Chai J, Shi Y. Oligomerization and activation of caspase-9 induced by Apaf-1 CARD. Proc Natl Acad Sci USA 2002; 99: 4197-4202.

80. Shi Y. Apoptosome: the cellular engine for the activation of caspase-9. Structure 2002; 10 : 285-288. 